\author{
What Does It Take to Get a Vegetarian to Eat Meat? \\ Factors Predicting Dietary Adherence \\ Daniel L. Rosenfeld \\ University of California, Los Angeles
}

\begin{abstract}
Author Note:
Daniel L. Rosenfeld, Department of Psychology, University of California, Los Angeles Correspondence concerning this article may be sent to Daniel L. Rosenfeld, Department of Psychology, University of California, Los Angeles, 1285 Franz Hall, Los Angeles, CA 90095, United States of America. E-mail address: rosenfeld@g.ucla.edu.
\end{abstract}




\begin{abstract}
Many people say they are vegetarian yet still eat meat on occasion. Despite this paradox having been documented extensively, multivariate attempts to explain individual differences in vegetarians' levels of dietary adherence are lacking. The current paper presents three highly powered studies $(N \mathrm{~s}=589,592$, and 594) that examined what psychological constructs predict a vegetarian's level of self-imposed dietary adherence, along with a meta-analysis (Study 4) of these studies. The meta-analysis indicated that the majority (54\%) of vegetarians were open to the possibility of eating meat. Consistently, factors that distinguished low-adherence from highadherence vegetarians were social identity variables related to vegetarianism, motivation for vegetarianism, disgust toward meat, and general liking of meat. Higher centrality of vegetarian dieting to one's identity, greater disgust toward meat, lower liking of meat, longer duration of following a vegetarian diet, and considering oneself to be a vegan were unique predictors of higher dietary adherence intention. Implications for theory, research methodology, and practice are discussed.
\end{abstract}

Keywords: vegetarianism, social identity, food choice, motivation, disgust 


\section{What Does It Take to Get a Vegetarian to Eat Meat? \\ Factors Predicting Dietary Adherence}

Many people say they are vegetarian yet still eat meat on occasion (Barr \& Chapman, 2002; Rothgerber, 2014a, 2017; Stiles, 1998; White, Seymour, \& Frank 1999; Willetts, 1997).

Despite the fact that eating meat fundamentally defies the definition of being a vegetarian, meateating vegetarians appear to comprise a substantial proportion of the vegetarian population: For example, a study by Kwan and Roth (2004) revealed that $40 \%$ of self-identified vegetarians actually eat meat. Although studies documenting this paradoxical discrepancy between selfidentifying as a vegetarian and following a strictly vegetarian diet are plentiful, far less work has explained within-group heterogeneity in dietary adherence - that is, why do some vegetarians follow their diets more strictly than other vegetarians do? In the current research, I sought to address this knowledge gap by identifying psychological constructs that predict a vegetarian's level of dietary adherence intention.

Studying dietary adherence among vegetarians is important for two main reasons. First, it can inform optimal methods of operationalizing vegetarianism — an often subjectively defined concept (Ruby, 2012). Divergences between whether people self-identify as vegetarian and whether they eat meat, even in small amounts, can obscure how demographers and researchers operationalize vegetarianism. If an individual considers him or herself to be a vegetarian, yet reports eating meat every now and then, should an investigator classify him or her as a vegetarian or a meat-eater? Understanding psychological factors that distinguish vegetarians who follow their diets strictly from those who allow themselves to eat some meat may provide insights into the tradeoffs one may encounter in operationalizing vegetarian status based on people's self-identifications versus their actual eating behaviors. 
Second, studying vegetarian dietary adherence can contribute insights from theoretical perspectives on social identity, motivation, and morality to the study of eating behavior. The recurrent finding that many people self-identify as vegetarian yet continue to eat meat has led some scholars to suggest that vegetarianism is an identity—-specifically, a social identity (Tajfel \& Turner, 1985)—beyond just a diet (Jabs et al., 2000; Rosenfeld \& Burrow, 2017a; Stiles, 1998). Evidently, one can label oneself as a vegetarian without following a vegetarian diet strictly. Psychological science is well poised to investigate the underpinnings of this diet-label paradox: Already, several studies have revealed useful insights into how people construct identity around vegetarian dieting, what motivates people to adopt a vegetarian diet, and how vegetarians moralize their food choices and develop feelings of disgust toward meat (Fessler, Arguello, Mekdara, \& Macias, 2003; Fox \& Ward, 2008a, 2008b; Rosenfeld, 2018, 2019c, 2019d; Rosenfeld \& Burrow, 2017a, 2017b; Rothgerber, 2014a, 2015; Rozin, Markwith, \& Stoess, 1997). Recent research has also linked these phenomena to dietary adherence, explaining how vegetarians' dietary motivations and feelings of disgust toward meat associate with how strictly they follow their diets (Rosenfeld, 2019d; Rothgerber, 2014a, 2015).

Still, however, two limitations characterize the current state of evidence on vegetarian dietary adherence. First, research has yet to conduct a comprehensive multivariate test of which factors uniquely explain variance in vegetarians' self-imposed standards of dietary adherence, in contrast to considering one or only a few factors simultaneously. Thus, there is the lingering concern of shared variance, such that it remains unknown which factors uniquely predict adherence and which factors are mere confounds. Second, research has yet to test whether social identity factors uniquely predict vegetarians' dietary adherence over and above other known 
predictors. The current research sought to address these knowledge gaps through three highly powered preregistered studies, along with a meta-analysis of these studies.

\section{Probable Predictors of Vegetarian Dietary Adherence}

As of yet, only a few studies have identified factors associated with varying levels of dietary adherence among vegetarians. One's motivation for eschewing meat has emerged as a significant predictor. Vegetarians who follow their diets for ethical reasons - namely, out of a concern for animal rights/welfare - adhere more strictly than health-motivated vegetarians do (Rosenfeld, 2019d; Rothgerber, 2014a). A probable reason for this link is that people who subscribe to vegetarianism out of ethical concerns for animals are more disgusted by meat than people who are vegetarian for either health or environmental reasons are (Rosenfeld, 2019d). Disgust toward meat is theoretically and evolutionarily sensible to influence dietary strictness, as disgust informs how people deem specific foods to be edible or inedible (Rozin \& Fallon, 1987). Many vegetarians, particularly those with moral concerns about animals, view meat as disgusting (Rosenfeld, 2019d; Rozin et al., 1997). Vegetarians who report feeling more disgusted by meat and view meat as less appealing (e.g., its taste, smell, texture, and appearance) are more likely to exhibit greater dietary adherence (Rothgerber, 2014a). Yet beyond vegetarians' reasons for following their diets and their sensory-affective attitudes toward meat, how they construct social identity around their diets likely influences their adherence as well.

\section{Social Identity Aspects of Vegetarianism}

According to social identity theory, people derive a sense of identity from each of their memberships in various social categories (Tajfel \& Turner, 1985). Although often treated as simply a dietary pattern, vegetarianism exemplifies a social category into which an individual who avoids eating meat may readily self-categorize - a category, furthermore, that deviates from 
social norms in most cultures (Rosenfeld \& Burrow, 2017a). A few studies have applied social identity perspectives in studying aspects of vegetarian dietary adherence, though only from an onlooker's perspective. For one, vegetarians - particularly those who identify strongly as vegetarian - are inclined to express negative attitudes toward other vegetarians who eat meat (Hornsey \& Jetten, 2003). Vegetarians' dietary motivations may even influence how they judge in-group dietary violations, such that ethically motivated vegetarians devalue other vegetarians' acts of eating meat more strongly than health-motivated vegetarians do (Rothgerber 2014b). Vegetarians perceive other vegetarians' dietary violations as threatening to their group's distinctiveness, existence, and message and as a stimulus for increasing individual temptation, all of which are factors that predict vegetarians' evaluations of other in-group members' dietary violations (Rothgerber, 2014b). What these findings highlight is that, in violating their diets, vegetarians become susceptible to rejection from their social group and can face social identity threats (Rosenfeld \& Burrow, 2017a).

As is the case with other social identities (Luhtanen \& Crocker, 1992; Tajfel \& Turner, 1985), people vary in the extent to which they view being vegetarian as a defining feature of their self-concept and in the emotional significance they attach to being vegetarian. Three constructs within vegetarian identity capture facets of this variance: centrality, private regard, and public regard (Rosenfeld and Burrow, 2017a). Theoretical perspectives suggest that these vegetarian identity dimensions may predict vegetarians' willingness to eat meat.

Centrality refers to the extent to which one views being vegetarian as a predominant feature of one's self-concept (Rosenfeld \& Burrow, 2017a). Vegetarians with a high centrality, that is, derive a large portion their identity from vegetarianism and are more inclined to view following their diets as intertwined with their self-esteem. Theoretical perspectives on self- 
consistency suggest that, across varying social contexts, people are motivated to behave in ways that align with their self-image (Lecky, 1945). Accordingly, I predicted that having higher vegetarian identity centrality would motivate vegetarians to maintain a consistent sense of self by adhering to their diets more strictly. By eating meat, vegetarians contradict their self-definition, and this contradiction would evoke a particularly strong feeling of self-inconsistency for vegetarians who identify strongly as vegetarian. Social identity theory (Tajfel \& Turner, 1985) and research on social identity threat (Branscombe, Ellemers, Spears, \& Doosje, 1999) similarly predict a positive link between vegetarian centrality and dietary adherence. Not only do people aim to act in accordance with norms of their social identities in order to promote in-group distinctiveness, but people may further face acceptance threat — having their position in their social group undermined - if they violate a norm that defines their social identity (Branscombe et al., 1999). Thus, vegetarians with greater identity centrality, who internalize being vegetarian as more core to their self-concept, are likely to experience harsher threats to their self-esteem if they violate their diets.

Whereas centrality characterizes the importance people place on an identity domain, private and public regards characterize specific forms of self-esteem people derive from an identity domain (Luhtanen \& Crocker, 1992; Rosenberg, Schooler, Schoenbach, \& Rosenberg, 1995). In the context of vegetarian identity, private regard refers to one's personal feelings toward other vegetarians and toward being vegetarian, whereas public regard refers to one's feelings about the way in which meat-eaters and the larger society view vegetarians, with both constructs operating in terms of positive-negative valence (Rosenfeld \& Burrow, 2017a). Private regard directly shapes one's self-esteem, as individuals seek to make identity domains of which they are proud more central to their overall self-concept in order to maintain a positive self- 
image (Stryker \& Serpe, 1982). Thus, higher private regard likely promotes dietary adherence among vegetarians. Vegetarians with higher private regard may more strongly desire to act in accordance with meat-abstention norms defining their vegetarian identity, as possessing that social identity enhances their sense of positive group affiliation and, in turn, their self-esteem.

Public regard may also have a positive relationship with adherence. Vegetarianism is a stigmatized identity associated with a range of negative stereotypes (Minson \& Monin, 2012). Internalizing these stereotypes may lead vegetarians to develop lower public regard, feeling stigmatized for their food choices (Rosenfeld \& Burrow, 2017a). Yet vegetarianism is a concealable identity, one that individuals may hide strategically through impression management (LeRette, 2014; Jabs et al., 2000; Rosenfeld \& Burrow, 2017a). Research on stigma and identity concealment suggests that vegetarians with lower public regard may feel a greater desire to conceal their vegetarian identity to avoid feeling stigmatized (Quinn \& Chaudoir, 2009). In social situations in which vegetarians anticipate diet-based stigma, they may conceal their vegetarian identity by eating meat. Among vegetarians with lower public regard, exercising a weaker dietary adherence may be an impression management strategy for avoiding stigma and maintaining one's self-esteem.

\section{Additional Considerations}

In order to isolate the unique predictive value of vegetarian social identity constructs, I concurrently considered four additional factors likely to associate with vegetarian dietary adherence: dietary goal orientation, duration, vegan status, and gender.

Vegetarian dietary goal orientations. Motivation constitutes an individual's goaloriented ambitions for following a vegetarian diet — that is, why he or she chooses to eschew meat (Rosenfeld \& Burrow, 2017b). Reviewing the literature on what reasons vegetarians report 
for eschewing meat, Rosenfeld and Burrow (2017a, 2017b, 2018) have theorized that three specific types of goal orientations involved in following a vegetarian diet include prosocial, personal, and moral orientations. Whereas prosocial orientation refers to aims to benefit a cause beyond oneself, personal orientation refers to aims to benefits oneself. Moral orientation refers to aims to abide by one's principles of right and wrong. Within-group heterogeneity in vegetarians' reasons for following their diets is substantial, as vegetarians vary greatly in their dietary goal orientations (Rosenfeld, 2019a, 2019c, 2019d; Rosenfeld \& Burrow, 2018). Evidence exists to suggest that prosocial, personal, and moral goal orientations may divergently predict vegetarians' levels of dietary adherence. I posited that feeling strong senses of prosocial and moral orientations, in particular, would propel vegetarians to adhere to their diets strictly.

Whereas personally oriented vegetarians principally see their diets as a means of improving their well-being, avoiding disease, or enhancing their spirituality, prosocially oriented vegetarians tend to construe their diets as a selfless pursuit, wherein commitments to helping animals or the environment may so much as take precedence over concerns about their own utility (Fox \& Ward, 2008a, 2008b; Rosenfeld \& Burrow, 2017a). Prosocially oriented vegetarianism, moreover, can embody a social movement, one that is often centered on promoting animal rights and/or environmentalism. Efforts to change societally dominant values (e.g., the pervasiveness of omnivorous dieting) through such movements are undermined when people advocate for the movement's message yet act in a contradictory manner (Hornsey \& Jetten, 2003). Vegetarians who eat meat may threaten their social group's authenticity and undercut its defining message that people ought not to eat meat (Rothgerber, 2014b). As such, prosocial goal orientation likely constitutes a unique impetus for adhering strictly to one's vegetarian dietary intentions. 
Some research also suggests that moral goal orientation is more strongly associated with dietary adherence than personal orientation is. For example, Ogden, Karim, Choudry, and Brown (2007) found that ethical motivation — but not health motivation—predicts successful exclusion of a food group from one's diet. Moreover, vegetarians committed to following their diets strictly are more likely to be ethically motivated than health-motivated (Rosenfeld, 2019d; Rothgerber, 2014b). Moralization of vegetarianism can elicit feelings of disgust toward meat, which may prompt vegetarians to follow their diets more strictly (Fessler et al., 2003; Rozin et al., 1997) Still, moral dietary goal orientation appears to offer unique predictive ability: Following a vegetarian diet out of moral concerns for animals has been found to predict dietary adherence among vegetarians over and above disgust toward meat (Rosenfeld, 2019d). These findings support notions that morality is central to most people's self-concepts (Monin, 2007), as ethical motivation appears to promote dietary self-consistency more than health motivation does. By following their diets more strictly, morally oriented vegetarians can maintain a positive selfimage, holding themselves in high moral regard and avoiding moral dissonance. To eat meat would mean to violate their moral code. As such, I predicted that vegetarians with a strong moral orientation would be more resistant to eating meat.

Duration. Duration refers to the amount of time for which one has been a vegetarian (Rosenfeld \& Burrow, 2017a). What makes vegetarianism a unique identity domain is that most people voluntarily adopt it later in life, deciding to give up meat after having been a meat-eater of many years (Beardsworth \& Keil, 1991). If vegetarians internalize their diets as a defining social identity (Rosenfeld \& Burrow, 2017a), and social identities collectively make up a portion of one's self-concept (Tajfel \& Turner, 1985), then people who maintain a vegetarian diet for a longer duration may feel a greater sense of self-inconsistency if they were to eat meat — it would 
contradict a temporally stable feature of who they are and who they have been. Indeed, Rothgerber (2014a) found that, compared to vegetarians who would consider eating meat, vegetarians who would refuse to eat meat reported having been following their diets for a longer time. What remains untested, however, is duration's unique predictive ability for adherence.

Vegan status. Whether or not one is a vegan may be important to consider. Vegans eschew not only meat but also other animal products such as egg and dairy. Because they follow a type of vegetarian diet, vegans are conceived conventionally as a subgroup of vegetarians (Beardsworth \& Keil, 1992; Rosenfeld \& Burrow, 2017a; Ruby, 2012), with the term "vegetarians" referring to both vegetarians and vegans, inclusively. Still, it may be useful to test whether individuals' statuses as vegan or non-vegan vegetarian predicts dietary adherence, particularly in light of a social identity lens attentive to such nuanced categorical distinctions. For example, compared to other vegetarians, vegans express greater concerns about animal rights, animal welfare, and animal experimentation; devalue in-group dietary violations more strongly; and exhibit higher dietary identity centrality, higher private regard, and lower public regard (Izmirli \& Phillips, 2011; Kessler et al., 2016; Lund et al., 2016; Rosenfeld, 2019a; Rothgerber 2014b).

Gender. A final additional factor to consider is gender, as meat is strongly associated with masculinity (Sobal, 2005). Women are more likely than men are to go vegetarian (Rosenfeld, 2018), and men and women express their vegetarian identities in different ways, with women adhering to vegetarianism more strictly than men do (Rosenfeld, 2019b). Not only do meat-eaters exhibit greater biases against vegetarian men than against vegetarian women (MacInnis \& Hodson, 2017), but vegetarian men even face more adverse social consequences for following their diets than vegetarian women do (Sedupane, 2017; Torti, 2017). Given that men 
and women have different experiences with respect to vegetarian dieting, they may exhibit varying degrees of willingness to violate their diets.

\section{Overview of the Current Research}

Rosenfeld and Burrow (2017a) posit that vegetarians require a sufficient feeling of control over their food choices in order to follow their dietary patterns successfully. When physiological or social pressures to eat meat arise, an individual's feeling of control can diminish as willingness to eat meat can increase. As a result, an individual's self-imposed level of dietary strictness becomes relevant. Situations that are likely to pressure individuals to eat meat include those in which eating meat is socially endorsed and constitutes an evident social norm, meatless food options are scarce, social evaluation is heightened, or perhaps even explicit rewards (e.g., monetary payments) are incentivized for meat consumption. In this research, I examined vegetarians' behavioral inclinations across diversified conditions that tested the boundaries of their feeling of control, focusing on multiple types of meats. Namely, when pressures to eat meat arise, which psychological constructs may keep a vegetarian on course with intentions to eschew meat? Synthesizing the predictions outlined in the sections above, my hypotheses throughout this paper's studies were that higher vegetarian identity centrality, higher vegetarian identity private regard, higher vegetarian identity public regard, higher prosocial orientation, higher moral orientation, greater disgust toward meat, lower meat liking, and longer duration would predict higher dietary adherence.

In order to assess multiple facets of dietary adherence, I employed three varied paradigms through three surveys. In the first paradigm (Study 1), I measured the amount of money vegetarians reported they would need to get paid in order to eat meat. Receiving a financial incentive can motivate people to behave in undesired ways and may be a useful proxy for 
behavioral commitment. As meat-eaters not only tease vegetarians often (LeRette, 2014) but also express more favorable attitudes toward vegetarians who violate their diets than toward vegetarians who adhere strictly (Hornsey \& Jetten, 2003), one can imagine a vegetarian's meateating friends — almost comically_ offering him or her money to eat meat.

In the second paradigm (Study 2), I measured the likelihood of vegetarians ordering meat while dining with friends at a steakhouse renowned for its steak, where the only vegetarian option is a salad. Given that taste preference is a leading determinant of what foods people decide to eat (Furst, Connors, Sobal, Bisogni, \& Falk, 2000), including intentions to eat meat (Rosenfeld \& Tomiyama, 2020), it is unsurprising that some vegetarians decide to violate their diets due to cravings or missing the taste of meat (Willetts, 1997). Missing the taste of meat, likewise, is a leading reason as to why many vegetarians give up their diets altogether (Barr \& Chapman, 2002). Encountering the opportunity to eat a steak that is widely socially endorsed as delicious may be tempting for some vegetarians and consequently test their self-imposed level of dietary adherence.

In the third paradigm (Study 3), I measured the likelihood of vegetarians eating a chicken dish served to them while on a job interview. On a job interview, social evaluation is omnipresent, as interviewees aim to make a favorable impression on the interviewer. Eating behavior, as a highly social phenomenon, is strongly relevant to impression management, as people can strategically select foods that present a positive image of who they are (Vartanian, 2015). An interviewee's rejecting a food served to him or her at a meal may create a negative impression of the interviewee as inflexible and disagreeable - traits that are likely to impair the interviewer's impression of him or her (Graziano \& Eisenberg, 1997). Thus, on a job interview, a vegetarian who is served meat may feel a heightened sense of pressure to eat it in order to appear 
agreeable. Ultimately, through these varied paradigms, I sought to enhance my investigation's ecological validity, acquiring a close proxy for vegetarians' resistance to plausible pressures and their commitments to meat abstention.

\section{Study 1}

\section{Method}

This study's sample size, materials, and analyses were preregistered via the Open Science Framework (OSF) (see https://osf.io/n2p94/?view_only=c904a29d8c234bceaa30195c911150ba for preregistration).

Participants. A power analysis conducted using WebPower (available at https://webpower.psychstat.org/wiki/models/index) indicated that 308 participants would provide $90 \%$ power to detect a small-medium effect size of $f^{2}=0.08$ in a model with 15 predictorswhich is the number I planned this study's regression model to have in its final hierarchical stage — at a significance threshold of $p=.05$. Accordingly, in order to maximize the power of my study design further, I recruited a total of 600 participants.

Six hundred self-identified vegetarian participants from the United States were recruited to take part in this study via Amazon Mechanical Turk (MTurk). After excluding three participants who reported that they were not vegetarian/vegan, four participants who failed an attention check in the survey, and four participants who reported a non-binary gender identity status, 589 participants ( $57 \%$ female) between the ages of 18 and $81\left(M_{\text {age }}=35.22, S D=11.16\right)$ were retained for analyses.

\section{Materials.}

Vegetarian identity centrality and regards. Vegetarian identity centrality, private regard, and public regard were assessed using Rosenfeld and Burrow's (2018) Dietarian Identity 
Questionnaire (DIQ) centrality, private regard, and public regard subscales (as validated for use among vegetarians by Rosenfeld, 2019c). These scales began with an initial item that assessed which of the following animal products participants eat or do not eat: red meat, poultry, fish, egg, and dairy. Below this item was a prompt highlighting that, for the rest of the survey, a participant's "dietary pattern" referred to those foods he or she indicated above. Following this dietary pattern item, participants completed the centrality, private regard, and public regard subscales. An example item for the 5-item centrality scale $(\alpha=.93)$ included "My dietary pattern defines a significant aspect of who I am." An example item for the 3 -item private regard scale $(\alpha$ $=.78$ ) included "People who follow my dietary pattern should take pride in their food choices." An example item for the 3-item public regard scale $(\alpha=.92)$ included "Following my dietary pattern is associated with negative stereotypes" (reverse-scored). Responses to all items ranged from 1 (Strongly Disagree) to 7 (Strongly Agree).

Dietary goal orientations. Dietary goal orientations were assessed using Rosenfeld and Burrow's (2018) DIQ prosocial, personal, and moral motivational orientations subscales. An example item for the 6-item prosocial orientation scale $(\alpha=.95)$ included "Concerns about social issues motivate me to follow my dietary pattern." An example item for the 3-item personal orientation scale $(\alpha=.89)$ included "I follow my dietary pattern because I am concerned about the effects of my food choices on my own well-being." An example item for the 3-item moral orientation scale $(\alpha=.92)$ included "I follow my dietary pattern because eating this way is the morally right thing to do." Responses to all items ranged from 1 (Strongly Disagree) to 7 (Strongly Agree).

Disgust toward meat. Disgust toward meat was assessed using a 4-item scale $(\alpha=.89)$ adapted from Rozin et al. (1997), as used in Rosenfeld (2019d). Two examples items were, "The 
thought of eating meat makes me nauseous" and "Eating meat is repulsive or disgusting." Items were measured via a scale of 1 (Strongly Disagree) to 7 (Strongly Agree).

Meat liking. Meat liking was assessed using a 4-item scale $(\alpha=.95)$ adapted from Rothgerber (2014a). Through these items, participants reported how much they like the taste, smell, texture, and appearance of meat, with each item structured as "I like the [taste / smell / texture / appearance] of meat.” Responses to all items ranged from 1 (Strongly Disagree) to 7 (Strongly Agree).

Money to eat meat. The amount of money participants would need to get paid to get them to eat meat was assessed with the following question: "What is the smallest amount of money someone would need to pay you to get you to eat meat?" The response scale to this question began with "For me to eat a bite of meat, I would need to get paid at least:" and followed with the following responses: "\$0 - I would eat meat for any amount of money," "\$20," "\$50," “\$100," “\$250," “\$500," “\$1,000," “\$10,000," “\$100,000,” and “N/A - I refuse to eat meat for any amount of money." These responses were coded on a scale from 1 to 10 in this order.

Procedure. After consenting to take part in this research, participants reported their duration and whether they consider themselves, specifically, to be vegetarian or vegan. Then, participants indicated their dietary pattern. Next, participants completed the centrality, regards, goal orientations, disgust toward meat, and meat liking scales in a randomized order. Next, participants reported their minimum payment needed to eat a bite of meat. Lastly, participants completed demographic questions.

\section{Results and Discussion}

Data and analysis scripts are available at https://osf.io/khmz2/?view_only=f791f94406cd45dc89d1645f345fcd6d. 
First, I conducted bivariate correlations to test which social identity (centrality, private regard, and public regard), dietary goal orientation (prosocial, personal, and moral), and attitude toward meat (disgust and liking) variables were associated with amount of money needed to get paid to eat meat. Results indicated that vegetarians who would need to be paid higher amounts of money to eat meat - i.e., adhere to their diets more strictly — exhibited higher vegetarian identity centrality, higher vegetarian identity private regard, lower vegetarian identity public regard, higher prosocial orientation, higher moral orientation, greater disgust toward meat, and less meat liking (see Table 1). All of the directions of these correlations, except for that between amount of money and public regard, were consistent with hypotheses. The only variable tested that did not correlate significantly with amount of money needed to eat meat was personal orientation. Assumptions of ordinary least squares (OLS) regression were met, and hierarchical multiple OLS regression analysis revealed that, when controlling for all other variables in the full model (Step 3), higher vegetarian identity centrality, higher vegetarian identity private regard, greater disgust toward meat, lower meat liking, vegan status, and longer duration of following a vegetarian diet significantly predicted that participants would need to get paid a higher amount of money to eat meat (see Table 2). Variance inflation factors (VIFs) for all predictors in the full model were less than 3 , thus indicating that multicollinearity was not problematic (Akinwande, Dikko, \& Samson, 2015). 


\begin{tabular}{|lll|}
\hline & $r$ & $p$ \\
\hline Centrality & .36 & $<.001$ \\
Private Regard & .28 & $<.001$ \\
Public Regard & -.11 & .007 \\
Prosocial Orientation & .29 & $<.001$ \\
Personal Orientation & -.01 & .858 \\
Moral Orientation & .39 & $<.001$ \\
Disgust Toward Meat & .57 & $<.001$ \\
Meat Liking & -.50 & $<.001$ \\
\hline
\end{tabular}

Table 1: Bivariate correlations between main predictor variables of interest and amount of money needed to get paid to eat meat (Study 1). 


\begin{tabular}{|c|c|c|c|}
\hline & $\beta$ & $\mathrm{R}^{2}$ & $p$ \\
\hline Step 1 & & .20 & \\
\hline Centrality & $0.21^{* * *}$ & & $<.001$ \\
\hline Private Regard & $0.12^{* *}$ & & .005 \\
\hline Public Regard & -0.03 & & .382 \\
\hline Prosocial Orientation & -0.03 & & .575 \\
\hline Personal Orientation & $-0.10^{* *}$ & & .009 \\
\hline Moral Orientation & $0.25^{* * *}$ & & $<.001$ \\
\hline Step 2 & & .40 & \\
\hline Centrality & $0.09^{*}$ & & .022 \\
\hline Private Regard & $0.09^{*}$ & & .018 \\
\hline Public Regard & 0.00 & & .962 \\
\hline Prosocial Orientation & -0.03 & & .480 \\
\hline Personal Orientation & -0.06 & & .070 \\
\hline Moral Orientation & 0.08 & & .133 \\
\hline Disgust Toward Meat & $0.32^{* * *}$ & & $<.001$ \\
\hline Meat Liking & $-0.27^{* * *}$ & & $<.001$ \\
\hline Step 3 & & .42 & \\
\hline Centrality & $0.08^{*}$ & & .041 \\
\hline Private Regard & $0.09^{*}$ & & .022 \\
\hline Public Regard & 0.01 & & .825 \\
\hline Prosocial Orientation & 0.00 & & .978 \\
\hline Personal Orientation & -0.06 & & .088 \\
\hline Moral Orientation & 0.04 & & .400 \\
\hline Disgust Toward Meat & $0.28^{* * *}$ & & $<.001$ \\
\hline Meat Liking & $-0.25^{* * *}$ & & $<.001$ \\
\hline Duration & $0.13^{* * *}$ & & $<.001$ \\
\hline Vegan Status & $0.08^{*}$ & & .012 \\
\hline Gender & -0.02 & & .463 \\
\hline Race & 0.06 & & .067 \\
\hline Income & 0.00 & & .906 \\
\hline Education & -0.05 & & .145 \\
\hline Age & 0.00 & & .986 \\
\hline
\end{tabular}

Table 2: Hierarchical multiple OLS regression predicting amount of money needed to get paid to eat meat (Study 1).

Study 2

\section{Method}


This study's sample size, materials, and analyses were preregistered via OSF (see https://osf.io/uyfdq/?view_only=00d20463773a4034ab815cadf322592b for preregistration).

Participants. In Study 1, the full regression model with all predictors explained $42 \%$ of the variance in amount of money needed to eat meat, which equates to an effect size of $f^{2}=0.72$. A power analysis conducted using WebPower indicated that 47 participants would provide $90 \%$ power to detect this effect size in this full regression model. To maximize the power of my study design further to detect individual coefficients of smaller effects, and to be consistent with Study 1's methodology, I again recruited a total of 600 participants.

Six hundred self-identified vegetarian participants from the U.S. were recruited to take part in this study via MTurk. After excluding two participants who reported that they were not vegetarian/vegan, two participants who failed an attention check in the survey, and four participants who reported a non-binary gender identity status, 592 participants ( $57 \%$ female) between the ages of 18 and $77\left(M_{\mathrm{age}}=34.45, S D=10.35\right)$ were retained for analyses.

\section{Materials.}

Vegetarian identity centrality and regards. Vegetarian identity centrality, private regard, and public regard were assessed using the same methodology outlined in Study 1. Internal consistencies were high for all three of these scales (centrality $\alpha=.93$, private regard $\alpha=.79$, and public regard $\alpha=.92$ ).

Dietary goal orientations. Dietary goal orientations were assessed using the same methodology outlined in Study 1. Internal consistencies were high for all three of these scales (prosocial orientation $\alpha=.95$, personal orientation $\alpha=.86$, and moral orientation $\alpha=.92$ ).

Disgust toward meat. Disgust toward meat $(\alpha=.88)$ was assessed using the same methodology outlined in Study 1. 
Meat liking. Meat liking $(\alpha=.93)$ was assessed using the same methodology outlined in Study 1.

Likelihood of eating meat at a renowned steakhouse. The following vignette described a situation in which participants could imagine themselves dining at a steakhouse renowned for its steak, where the only vegetarian option is a measly salad: "Imagine that you are out to dinner with a few friends, preparing to order. You happen to be eating at a steakhouse that is rated as having the best steak in the entire state. The restaurant's only vegetarian option is a salad made up of just lettuce, tomatoes, and onions."

After reading this vignette, participants were asked, "In this scenario, how likely would you be to order steak for dinner?" to which responses ranged from 1 (I definitely would not order steak) to 5 (I definitely would order steak). This variable was reverse-coded so that a higher score would indicate higher dietary adherence, as to be consistent with the interpretation of Study 1's dietary adherence outcome.

Procedure. Procedure paralleled that of Study 1, with participants in this study completing the steakhouse thought experiment where participants in Study 1 reported their payment needed to eat meat.

\section{Results and Discussion}

Data and analysis scripts are available at https://osf.io/depqh/?view_only=9314e6bfb4ed4162889396a7915c9a67.

Because the outcome variable — likelihood of eating meat — was highly skewed (skewness $=-1.72$ ) such that a high proportion of participants selected the highest-scored response of "I definitely would not order steak," I transformed this continuous variable into a dichotomous variable that represented whether or not participants would consider eating meat. This 
transformation led the current study to deviate from its preregistration (which assumed a continuous outcome) but was necessary to analyze the data most appropriately, given that evident non-normality of residuals violated an assumption of OLS regression. The lower-scored four responses - which read, "I probably would not order steak," "It would be a close decision, but I might order steak," "I probably would order steak," and "I definitely would order steak"were all scored as 0 (i.e., low dietary adherence), whereas the highest-scored response of "I definitely would not order steak" was scored as 1 (i.e., high dietary adherence). Along this dichotomous variable, 207 participants (35\%) were classified as having a low dietary adherence whereas 385 (65\%) participants were classified as having a high dietary adherence.

Instead of bivariate correlations (as specified in preregistration, which assumed the use of OLS regression), I adapted analyses to fit the dichotomization of this study's outcome by conducting independent samples t-tests - using Welch-adjusted t-tests when variances were unequal — to test which main variables of interest were associated with likelihood of eating meat. Specifically, I compared these variables between vegetarians who would consider ordering steak (low adherence) with those who would definitely not order steak (high adherence). Significant differences between low-adherence and high-adherence vegetarians were observed for all variables except for personal orientation (see Table 3). These t-tests replicated the results of all bivariate correlations conducted in Study 1: Consistent with hypotheses set for bivariate correlations, compared to low-adherence vegetarians, high-adherence vegetarians exhibited higher vegetarian identity centrality, higher vegetarian identity private regard, higher prosocial orientation, higher moral orientation, greater disgust toward meat, and less meat liking. Inconsistent with hypotheses, again, is that high-adherence vegetarians reported lower public regard than low-adherence vegetarians did. Hierarchical multiple logistic regression analysis 
revealed that, when controlling for all other variables in the full model (Step 3), greater disgust toward meat, lower meat liking, vegan status, and longer duration of following a vegetarian diet significantly predicted that participants would refrain from order steak (i.e., exercise high dietary adherence) (see Table 4). VIFs for all predictors in the full model were less than 3, thus indicating that multicollinearity was not problematic.

\begin{tabular}{|c|c|c|c|c|c|c|}
\hline Variable & $\begin{array}{l}\frac{\text { Low Adherence }}{(n=207)} \\
\text { Mean (SD) }\end{array}$ & $\begin{array}{l}\frac{\text { High Adherence }}{(n=385)} \\
\text { Mean (SD) }\end{array}$ & $95 \%$ CI of Difference & $t$ & $d$ & $p$ \\
\hline Centrality & $4.55(1.43)$ & $5.18(1.27)$ & {$[-0.85,-0.40]$} & $5.44^{* * *}$ & 0.47 & $<.001$ \\
\hline Private Regard & $5.04(1.01)$ & $5.28(0.98)$ & {$[-0.41,-0.08]$} & $2.87^{* *}$ & 0.24 & .004 \\
\hline Public Regard & $3.86(1.44)$ & $3.59(1.53)$ & {$[0.01,0.52]$} & $2.06^{*}$ & 0.18 & .040 \\
\hline Prosocial Orientation & $4.19(1.66)$ & $4.89(1.47)$ & {$[-0.96,-0.44]$} & $5.29^{* * *}$ & 0.45 & $<.001$ \\
\hline Personal Orientation & $5.59(1.17)$ & $5.71(1.14)$ & {$[-0.32,0.07]$} & 1.29 & 0.10 & 197 \\
\hline Moral Orientation & $3.70(1.69)$ & $4.79(1.64)$ & {$[-1.37,-0.81]$} & $7.60^{* * *}$ & 0.65 & $<.001$ \\
\hline Disgust Toward Meat & $3.01(1.47)$ & $4.69(1.79)$ & {$[-1.94,-1.40]$} & $12.20^{* * *}$ & 1.03 & $<.001$ \\
\hline Meat Liking & $4.10(1.62)$ & $2.55(1.67)$ & {$[1.27,1.83]$} & $10.88^{* * *}$ & 0.94 & $<.001$ \\
\hline
\end{tabular}

Table 3: Differences between vegetarians who would consider ordering steak (low adherence) with those who would definitely not order steak (high adherence) along main variables of interest (Study 2). 


\begin{tabular}{|c|c|c|c|}
\hline & Odds Ratio & $\mathrm{R}^{2}$ & $p$ \\
\hline Step 1 & & .07 & \\
\hline Centrality & 1.15 & & .099 \\
\hline Private Regard & 0.98 & & .851 \\
\hline Public Regard & 0.96 & & .510 \\
\hline Prosocial Orientation & 0.99 & & .881 \\
\hline Personal Orientation & 1.03 & & .756 \\
\hline Moral Orientation & $1.38^{* * *}$ & & $<.001$ \\
\hline Step 2 & & .20 & \\
\hline Centrality & 1.03 & & .718 \\
\hline Private Regard & 0.93 & & .543 \\
\hline Public Regard & 1.01 & & .913 \\
\hline Prosocial Orientation & 1.01 & & .918 \\
\hline Personal Orientation & 1.16 & & .119 \\
\hline Moral Orientation & 1.13 & & .184 \\
\hline Disgust Toward Meat & $1.40^{* * *}$ & & $<.001$ \\
\hline Meat Liking & $0.71^{* * *}$ & & $<.001$ \\
\hline Step 3 & & .25 & \\
\hline Centrality & 0.99 & & .890 \\
\hline Private Regard & 0.88 & & .326 \\
\hline Public Regard & 1.03 & & .720 \\
\hline Prosocial Orientation & 1.06 & & .524 \\
\hline Personal Orientation & 1.16 & & .162 \\
\hline Moral Orientation & 1.14 & & .172 \\
\hline Disgust Toward Meat & $1.34^{* * *}$ & & $<.001$ \\
\hline Meat Liking & $0.72^{* * *}$ & & $<.001$ \\
\hline Duration & $1.40^{* * *}$ & & $<.001$ \\
\hline Vegan Status & $2.95^{* *}$ & & .002 \\
\hline Gender & 1.03 & & .901 \\
\hline Race & 0.94 & & .448 \\
\hline Income & 1.17 & & .110 \\
\hline Education & 0.90 & & .270 \\
\hline Age & 1.02 & & .105 \\
\hline
\end{tabular}

Table 4: Hierarchical multiple logistic regression predicting likelihood of ordering steak while dining at a steakhouse renowned for its steak (Study 2).

\section{Study 3}

As noted, the dichotomization of the outcome and according uses of t-tests and logistic regression in Study 2 deviated from preregistered analysis plans. In this Study 3, anticipating the 
potential for a similar need to dichotomize the outcome variable, I preregistered this decision contingent upon observing a highly skewed distribution. Through this, I aimed to conceptually replicate Study 2 in a more methodologically rigorous and controlled manner.

\section{Method}

This study's sample size, materials, and analyses were preregistered via OSF (see https://osf.io/h4nkb/?view_only=97b5777a329d40dabe8958d3d35dacf7 for preregistration).

Participants. To maintain the power of my study design above $90 \%$, and to be consistent with the methodology of Studies 1 and 2, I recruited a total of 600 self-identified vegetarian participants from the U.S. via MTurk. After excluding four participants who reported that they were not vegetarian/vegan, one participant who reported a non-binary gender identity status, and one participant who reported being younger than 18 years of age, 594 participants (58\% female) between the ages of 18 and $74\left(M_{\text {age }}=34.33, S D=10.35\right)$ were retained for analyses.

\section{Materials.}

Vegetarian identity centrality and regards. Vegetarian identity centrality, private regard, and public regard were assessed using the same methodology as in Studies 1 and 2. Internal consistencies were high for all three of these scales (centrality $\alpha=.93$, private regard $\alpha=.81$, and public regard $\alpha=.91)$.

Dietary goal orientations. Dietary goal orientations were assessed using the same methodology as in Studies 1 and 2. Internal consistencies were high for all three of these scales (prosocial orientation $\alpha=.95$, personal orientation $\alpha=.89$, and moral orientation $\alpha=.92$ ).

Disgust toward meat. Disgust toward meat $(\alpha=.87)$ was assessed using the same methodology as in Studies 1 and 2. 
Meat liking. Meat liking $(\alpha=.93)$ was assessed using the same methodology as in Studies 1 and 2.

Likelihood of eating meat on a job interview. The following vignette described a situation in which participants could imagine themselves being served meat while on an interview: "Imagine that you are out to dinner on an interview for your dream job. The interviewer forgot to ask about your dietary preferences beforehand and assumed that grilled chicken would be a good choice. So, earlier that day, the interviewer put in an order with the restaurant for both of you to have grilled chicken. When the food arrives, you realize that you have two options - either you can eat the chicken in front of you, or you can send the food back and order another dish."

After reading this vignette, participants were asked, “At this interview, how likely would you be to eat the grilled chicken?" to which responses ranged from 1 (I definitely would not eat the chicken) to 5 (I definitely would eat the chicken). This variable was reverse-coded so that a higher score would indicate higher dietary adherence, as to be consistent with Studies 1 and 2's dietary adherence outcomes.

Procedure. Procedure paralleled those of Studies 1 and 2, with participants in this study completing the job interview thought experiment where participants in Studies 1 and 2 reported their payment needed to eat meat and completed the steakhouse thought experiment, respectively.

\section{Results and Discussion}

Data and analysis scripts are available at https://osf.io/d5m46/?view_only=18b779434a30419d8551520c612f2f30. 
As specified in preregistration, because the outcome variable-likelihood of eating meat — was highly skewed (skewness $=-0.99)$ such that a high proportion of participants selected the highest-scored response of "I definitely would not eat the chicken," in turn leading to nonnormality of residuals, I transformed this continuous variable into a dichotomous variable that represented whether or not participants would consider eating meat, just as in Study 2. The lower-scored four responses_-which read, "I probably would not eat the chicken," "It would be a close decision, but I might eat the chicken," "I probably would eat the chicken," and "I definitely would eat the chicken"-were all scored as 0 (i.e., low dietary adherence), whereas the highestscored response of "I definitely would not eat the chicken" was scored as 1 (i.e., high dietary adherence). Along this dichotomous variable, 301 participants (51\%) were classified as having a low dietary adherence whereas 293 participants (49\%) were classified as having a high dietary adherence.

I conducted independent samples t-tests - using Welch-adjusted t-tests when variances were unequal - to test which main variables of interest were associated with likelihood of eating meat. Specifically, I compared these variables between vegetarians who would consider eating chicken (low adherence) with those who would definitely not eat chicken (high adherence). Significant differences between low-adherence and high-adherence vegetarians were observed for all variables except for public regard (see Table 5). Consistent with hypotheses, compared to low-adherence vegetarians, high-adherence vegetarians reported higher vegetarian identity centrality, higher vegetarian identity private regard, higher prosocial orientation, higher moral orientation, greater disgust toward meat, and less meat liking. High-adherence vegetarians also reported higher personal orientation. Results from a hierarchical multiple logistic regression analysis mirrored those obtained in Study 2: When controlling for all other variables in the full 
model (Step 3), greater disgust toward meat, lower meat liking, vegan status, and longer duration of following a vegetarian diet significantly predicted that participants would refrain from eating chicken (i.e., exercise high dietary adherence) (see Table 6). VIFs for all predictors in the full model were less than 3 , thus indicating that multicollinearity was not problematic.

\begin{tabular}{|c|c|c|c|c|c|c|}
\hline \multirow[t]{2}{*}{ Variable } & $\begin{array}{l}\text { Low Adherence } \\
\underline{(n=301)}\end{array}$ & $\begin{array}{l}\text { High Adherence } \\
\underline{(n=293)}\end{array}$ & $95 \%$ CI of Difference & $t$ & $d$ & \multirow[t]{2}{*}{$p$} \\
\hline & Mean (SD) & Mean (SD) & & & & \\
\hline Centrality & $4.55(1.30)$ & $5.26(1.27)$ & {$[-0.92,-0.51]$} & $6.76^{* * *}$ & 0.55 & $<.001$ \\
\hline Private Regard & $4.97(0.97)$ & $5.34(1.00)$ & {$[4.97,5.34]$} & $4.56^{* * *}$ & 0.38 & $<.001$ \\
\hline Public Regard & $3.71(1.37)$ & $3.64(1.56)$ & {$[3.71,3.64]$} & 0.58 & 0.05 & .559 \\
\hline Prosocial Orientation & $4.37(1.51)$ & $4.95(1.45)$ & {$[-0.81,-0.34]$} & $4.73^{* * *}$ & 0.39 & $<.001$ \\
\hline Personal Orientation & $5.53(1.17)$ & $5.73(1.17)$ & {$[-0.39,-0.01]$} & $2.11^{*}$ & 0.17 & .035 \\
\hline Moral Orientation & $4.05(1.64)$ & $4.71(1.69)$ & {$[-0.92,-0.39]$} & $4.80^{* * *}$ & 0.40 & $<.001$ \\
\hline Disgust Toward Meat & $3.46(1.55)$ & $4.91(1.75)$ & {$[-1.72,-1.18]$} & $10.65^{* * *}$ & 0.88 & $<.001$ \\
\hline Meat Liking & $3.73(1.69)$ & $2.47(1.66)$ & {$[3.73,2.47]$} & $9.17^{* * *}$ & 0.75 & $<.001$ \\
\hline
\end{tabular}

Table 5: Differences between vegetarians who would consider eating chicken (low adherence) with those who would definitely not eat chicken (high adherence) along main variables of interest (Study 3). 


\begin{tabular}{|c|c|c|c|}
\hline & Odds Ratio & $\mathrm{R}^{2}$ & $p$ \\
\hline Step 1 & & .06 & \\
\hline Centrality & $1.39^{* * * *}$ & & $<.001$ \\
\hline Private Regard & 1.18 & & .110 \\
\hline Public Regard & 1.01 & & .808 \\
\hline Prosocial Orientation & 1.06 & & .515 \\
\hline Personal Orientation & 0.97 & & .714 \\
\hline Moral Orientation & 1.04 & & .575 \\
\hline Step 2 & & .16 & \\
\hline Centrality & $1.22^{*}$ & & .041 \\
\hline Private Regard & 1.14 & & .235 \\
\hline Public Regard & 1.06 & & .399 \\
\hline Prosocial Orientation & 1.00 & & .980 \\
\hline Personal Orientation & 1.02 & & .804 \\
\hline Moral Orientation & 0.91 & & .284 \\
\hline Disgust Toward Meat & $1.44^{* * *}$ & & $<.001$ \\
\hline Meat Liking & $0.77^{* * *}$ & & $<.001$ \\
\hline Step 3 & & .19 & \\
\hline Centrality & 1.18 & & .094 \\
\hline Private Regard & 1.21 & & .105 \\
\hline Public Regard & 1.07 & & .324 \\
\hline Prosocial Orientation & 1.00 & & .974 \\
\hline Personal Orientation & 1.06 & & .509 \\
\hline Moral Orientation & 0.92 & & .370 \\
\hline Disgust Toward Meat & $1.40^{* * *}$ & & $<.001$ \\
\hline Meat Liking & $0.79^{* * *}$ & & $<.001$ \\
\hline Duration & $1.29^{* * *}$ & & $<.001$ \\
\hline Vegan Status & $2.01^{* *}$ & & .009 \\
\hline Gender & 0.82 & & .327 \\
\hline Race & 1.02 & & .789 \\
\hline Income & 1.07 & & .366 \\
\hline Education & 0.85 & & .052 \\
\hline Age & 1.00 & & .798 \\
\hline
\end{tabular}

Table 6: Hierarchical multiple logistic regression predicting likelihood of eating chicken when served a chicken dish while on a job interview (Study 3).

\section{Study 4: Meta-Analysis of Studies 1, 2, and 3}

Dietary adherence is a latent construct — a behavioral inclination to follow one's diet and a level of resistance one exercises in response to sensory and psychological pressures to eat meat. 
The above studies operationalized adherence through three outcomes: amount of money needed to get paid to eat meat, likelihood of eating meat at a renowned steakhouse, and likelihood of eating meat when served meat on a job interview. In order to identify which factors are predictors of dietary adherence across these paradigms with heightened statistical power, I standardized these outcomes into a common metric of dietary adherence and conducted a metaanalysis combining the data from the three studies reported above.

\section{Method}

Participants. This combined sample included 1,775 participants (57\% female) between the ages of 18 and $81\left(M_{\mathrm{age}}=34.67, S D=10.63\right)$.

Materials. Materials were those used in Studies 1 through 3. The only different material in this study was the dietary adherence variable. The dietary adherence variable in this study reflected participants' responses to the dietary adherence items in Studies 1 through 3 on a common metric. In order to operationalize dietary adherence on a common metric across studies, I created a dichotomous variable by coding participants who reported that they would consider eating meat (low adherence) as 0 and participants who reported that they would definitely not eat meat (high adherence) as 1. Participants coded as high adherence were those who refused to eat meat for any amount of money in Study 1 and those who definitely would not order meat in the scenarios in Studies 2 and 3. This coding method sorted participants into two similarly sized groups: 959 participants (54\% of sample) were considered to be low-adherence vegetarians, whereas 816 participants ( $46 \%$ of sample) were considered to be high-adherence vegetarians.

Procedure. The procedures for each of the three studies combined into this meta-analysis are outlined in their respective Procedure sections above.

\section{Results and Discussion}


Data and analysis scripts are available at https://osf.io/tx8ea/?view_only=6bcb96ed017f4ad7bf96578d083bd819.

Hierarchical multiple logistic regression analysis revealed that, when controlling for all other variables in the full model (Step 3), higher vegetarian identity centrality, greater disgust toward meat, lower meat liking, vegan status, longer duration, and lower educational attainment significantly predicted that participants would adhere to their diets strictly (see Table 7). VIFs for all predictors in the full model were less than 3, thus indicating that multicollinearity was not problematic. 


\begin{tabular}{|c|c|c|c|}
\hline & Odds Ratio & $\mathrm{R}^{2}$ & $p$ \\
\hline Step 1 & & .06 & \\
\hline Centrality & $1.32^{* * * *}$ & & $<.001$ \\
\hline Private Regard & 0.98 & & .796 \\
\hline Public Regard & 0.97 & & .411 \\
\hline Prosocial Orientation & 1.00 & & .953 \\
\hline Personal Orientation & 1.01 & & .803 \\
\hline Moral Orientation & $1.21^{* * *}$ & & $<.001$ \\
\hline Step 2 & & .14 & \\
\hline Centrality & $1.19^{* * *}$ & & .001 \\
\hline Private Regard & 0.94 & & .329 \\
\hline Public Regard & 1.00 & & .993 \\
\hline Prosocial Orientation & 0.99 & & .889 \\
\hline Personal Orientation & 1.07 & & .156 \\
\hline Moral Orientation & 1.05 & & .355 \\
\hline Disgust Toward Meat & $1.30^{* * *}$ & & $<.001$ \\
\hline Meat Liking & $0.77^{* * *}$ & & $<.001$ \\
\hline Step 3 & & .16 & \\
\hline Centrality & $1.17^{* *}$ & & .004 \\
\hline Private Regard & 0.94 & & .389 \\
\hline Public Regard & 1.01 & & .710 \\
\hline Prosocial Orientation & 1.02 & & .753 \\
\hline Personal Orientation & 1.08 & & .135 \\
\hline Moral Orientation & 1.03 & & .498 \\
\hline Disgust Toward Meat & $1.25^{* * *}$ & & $<.001$ \\
\hline Meat Liking & $0.79 * * *$ & & $<.001$ \\
\hline Duration & $1.23^{* * *}$ & & $<.001$ \\
\hline Vegan Status & $1.78^{* * *}$ & & $<.001$ \\
\hline Gender & 0.93 & & .500 \\
\hline Race & 1.02 & & .683 \\
\hline Income & 1.04 & & .391 \\
\hline Education & $0.89^{*}$ & & .017 \\
\hline Age & 1.00 & & .406 \\
\hline
\end{tabular}

Table 7: Hierarchical multiple logistic regression predicting dietary adherence in a meta-analysis of Studies 1, 2, and 3 .

\section{General Discussion}

The current findings provide several insights into individual differences in dietary adherence among vegetarians. Consistently across all three studies, social identity variables 
related to vegetarianism, motivation (i.e., goal orientation) for vegetarianism, disgust toward meat, and general liking of meat distinguished low-adherence from high-adherence vegetarians. Relative to low-adherence vegetarians, vegetarians who were committed to following their diets strictly reported that they view being vegetarian as a more predominant feature of their selfconcept (higher vegetarian identity centrality), take more pride in being vegetarian (higher vegetarian identity private regard), are more prosocially and morally motivated to follow their diets, are more disgusted by meat, and view meat as less appealing. These differences, moreover, ranged from small-medium to large in effect size. Neither awareness of stigma tied to being vegetarian (vegetarian identity public regard) nor following a vegetarian diet to improve one's well-being (personal goal orientation) exhibited consistent associations with dietary adherence. Taken together, these findings suggest that low-adherence and high-adherence vegetarians exhibit largely different psychological profiles with respect to social identity aspects of vegetarianism, dietary motivations, and attitudes toward meat.

A meta-analysis of all three studies revealed that, when adjusting for all other factors, higher vegetarian identity centrality, greater disgust toward meat, lower liking of meat, longer duration of following a vegetarian diet, and considering oneself to be a vegan were significant predictors of higher commitment to dietary adherence.

\section{Implications for Theory}

Replicating and extending prior findings. These findings extend prior work (e.g., Rosenfeld, 2019d; Rothgerber, 2014a), providing novel evidence to suggest that how disgusted people feel by meat, how much they like meat, and for how long they have been vegetarian are not only bivariate correlates but also robust, unique predictors of vegetarian dietary adherence, over and above a host of other variables. First, the finding that disgust toward meat predicts strict 
meat avoidance among vegetarians supports Rozin and Fallon's (1987) theoretical conception of disgust as a food-related emotion that inhibits people's willingness to eat potentially contaminated objects, particularly ones of animal origin. Second, the finding that vegetarian dietary duration uniquely predicted dietary adherence suggests that eating meat may become less desirable as one follows a vegetarian diet for a longer period of time. One explanation for this link may be that vegetarianism becomes more temporally stable to one's self-concept over time, which may make violating one's diet a greater threat to maintaining a coherent self-image.

Social identity theory. Beyond its extensions of prior work on meat disgust, meat liking, and dietary duration, the current research adds that vegetarian identity centrality is a unique predictor of dietary adherence — one that had not been documented previously. The finding that identifying strongly as a vegetarian (i.e., higher centrality) predicts higher dietary adherence supports predictions derived from coupling social identity theory with theoretical perspectives on self-consistency. One inference that could be drawn from the current research is that adhering to one's diet strictly may be a means of maintaining a consistent sense of self, particularly among vegetarians with high centrality. That is, the act of eating meat starkly conflicts with selfidentifying as vegetarian, and the psychological discomfort resulting from this conflict may be amplified if being vegetarian is a core part of one's self-concept. Another mechanism at play may relate to social identity threat (Branscombe et al., 1999): Vegetarians with high centrality may experience harsher threats to their self-esteem if other vegetarians, or even meat-eaters, were to deny them vegetarian status - i.e., not accept them as "true" vegetarians - because they violated their diet.

Although qualitative studies conceiving vegetarianism as an identity are abundant (e.g., Fox \& Ward, 2008b; LeRette, 2014; Stiles, 1998; Yeh, 2014), quantitative research on social 
identity aspects of vegetarianism is profoundly lacking. The current paper is one of the first to document that the way in which vegetarians construct social identity around their diets offers unique predictive value for meaningful outcomes such as dietary adherence. Social identity theory has transformed the studies of race, ethnicity, gender, political orientation, and many more domains, and offers a promising perspective for studying eating behavior, particularly in the realm of vegetarianism.

Morality and disgust. The current research also has implications for the application of moral psychology to eating behavior. In the meta-analysis, moral orientation was a significant positive predictor of adherence in the first step of the hierarchical regression yet became nonsignificant in the second step when accounting for disgust toward meat and meat liking. As such, the link between moral orientation and dietary adherence may be attributed to one of these variables, most likely disgust. Prior studies have found that morally motivated vegetarians are more disgusted by meat than health-motivated vegetarians are (Jabs et al., 1998; Rothgerber, 2014a; Rozin et al., 1997), and recent evidence suggests that disgust toward meat may mediate the link between vegetarians' dietary motivations and dietary adherence (Rosenfeld, 2019d). What has yet to be documented, however, is causal evidence for this model— that feelings of disgust toward meat emerge out of moralizing vegetarianism and directly cause people to follow vegetarian diets strictly. Evidence suggests that it may not be moralization but, rather, consciously associating meat with its origins of coming from a formerly living animal that elicits meat disgust in vegetarians and promotes stringent dietary adherence (Rosenfeld, 2019d).

Further considering the causal relationships between moralization, animal-meat association, and disgust can be informative.

\section{Implications for Research Methodology}


As investigators design new studies, how should they first define what exactly makes someone a vegetarian? The meta-analyses of all studies revealed a statistic that many might find staggering: $54 \%$ of vegetarians reported that they are open to the possibility of eating meat. Thus, for many individuals, being a vegetarian may not be such a rigid dietary choice but rather a flexible one that individuals manage by constructing and negotiating social identity (Jabs et al., 2000; Rosenfeld \& Burrow, 2017a). As demographers estimate the prevalence of vegetarianism in populations and as researchers recruit vegetarian participants for studies, it may be advantageous to consider the notion that whether or not people self-identify as vegetarian and whether or not they report following a vegetarian (i.e., entirely meatless) diet may diverge. The way in which one operationalizes vegetarian status may be critical: Surveys that assess whether people self-identify as vegetarian (e.g., Gallup, 2018) or whether people follow a strictly vegetarian diet (e.g., The Vegetarian Resource Group, 2016) assess different constructs and yield different results. By understanding the conceptual underpinnings of this divergence and developing theoretically grounded models to predict dietary adherence systematically, investigators can reconcile the paradox between vegetarian dieting and self-identification more holistically and employ methodology more strategically.

\section{Applied Implications}

In addition to its basic and methodological implications for theory and future research, the current paper also offers applied implications for explaining and controlling eating behavior. Given that greater adoption of vegetarian diets offers an effective strategy for improving public health and promoting environmental sustainability (Willett et al., 2019), it is increasingly important to unravel what factors can enable people to adopt and maintain vegetarian diets successfully. Vegetarianism has an extraordinarily high attrition rate: Not only do many 
vegetarians violate their diets sporadically but an estimated $84 \%$ of vegetarians eventually give up their diets altogether and return to eating meat (Herzog, 2014). Although the current research is correlational in nature and thus does not allow for causal inference, it can nevertheless provide a starting point for considering what factors may enable vegetarians to adhere to their diets more successfully. Namely, finding ways to increase one's sense of vegetarian identity centrality; to view meat as more disgusting; and to dislike meat's taste, smell, texture, and appearance more strongly may confer protection against lapsing back to eating meat. Additional research is needed, however, to assess the causality of these relationships.

\section{Limitations}

Three limitations of the current research stem from its cross-sectional survey design. First, participants identified themselves as vegetarian, which may have led some participants who are truly non-vegetarians to misrepresent themselves. Although the divergence between self-identification as vegetarian and actual eating behavior is of central relevance to this paper, and of theoretical and practical interest, the potential remains that some participants lied in even self-identifying as vegetarian. Second, dietary adherence was operationalized via self-reported intentions, which, while indicative of participants' attitudes toward their dietary behavior, are not a perfect proxy for actual behavior. Third, there is potential for reverse causality, such that greater adherence to a vegetarian diet may alter individuals' scores on the current studies' predictor variables, rather than solely the reverse. For example, adhering strictly to a meatless diet may lead vegetarians to feel increasingly disgusted by the thought of eating meat. Longitudinal designs would be valuable to understand causes and effects of dietary adherence more clearly. A fourth limitation of this research is that it did not consider all variables that may explain differences in vegetarian adherence. Future research should also examine social network 
variables (e.g., number of vegetarian friends and family members, social support for vegetarianism) and relevant ideologies (e.g., political orientation, endorsement of carnism, endorsement of traditions/rituals).

\section{Conclusion}

The current set of studies suggest that higher centrality of being a vegetarian to one's overall self-concept, greater disgust toward meat, lower liking of meat, longer duration of following a vegetarian diet, and considering oneself to be a vegan predict that one seeks to adhere to one's meatless diet more strictly. Identifying these factors as significant predictors of dietary adherence not only advances theoretical perspectives on the links between social identity, morality, disgust, and food choice, but also informs best practices for promoting successful vegetarian eating patterns for the benefits of public health and environmental sustainability. 


\section{References}

Akinwande, M. O., Dikko, H. G., \& Samson, A. (2015). Variance inflation factor: As a condition for the inclusion of suppressor variable(s) in regression analysis. Open Journal of Statistics, 5, 754-767.

Barr, S. I., \& Chapman, G. E. (2002). Perceptions and practices of self-defined current vegetarian, former vegetarian, and nonvegetarian women. Journal of the American Dietetic Association, 102, 354-360.

Beardsworth, A. D., \& Keil, E. T. (1991). Vegetarianism, veganism, and meat avoidance: Recent trends and findings. British Food Journal, 93, 19-24.

Beardsworth, A. D., \& Keil, E. T. (1992). The vegetarian option: Varieties, conversions, motives and careers. The Sociological Review, 40, 253-293.

Branscombe, N. R., Ellemers, N., Spears, R., \& Doosje, B. (1999). The context and content of social identity threat. In N. Ellemers, R. Spears, \& B. Doosje (Eds.), Social identity: Context, commitment, content (pp. 35-58). Oxford, England: Blackwell.

Fessler, D. M., Arguello, A. P., Mekdara, J. M., \& Macias, R. (2003). Disgust sensitivity and meat consumption: A test of an emotivist account of moral vegetarianism. Appetite, 41, $31-41$.

Fox, N., \& Ward, K.J. (2008a). Health, ethics and environment: A qualitative study of vegetarian motivations. Appetite, 50, 422-429.

Fox, N., \& Ward, K. J. (2008b). You are what you eat? Vegetarianism, health and identity. Social Science \& Medicine, 66, 2585-2595.

Furst, T., Connors, M., Sobal, J., Bisogni, C., \& Falk, L. W. (2000). Food classifications: Levels and categories. Ecology of Food and Nutrition, 39, 331-355. 
Gallup. (2018, August 1). Snapshot: Few Americans vegetarian or vegan. Gallup. Retrieved from https://news.gallup.com/poll/238328/snapshot-few-americans-vegetarianvegan.aspx?g_source=link_NEWSV9\&g_medium=NEWSFEED\&g_campaign=item_\&g _content=Snapshot $\% 3 \mathrm{a} \% 2520 \mathrm{Few} \% 2520$ Americans $\% 2520$ Vegetarian $\% 2520$ or $\% 2520 \mathrm{~V}$ egan

Graziano, W. G., \& Eisenberg, N. H. (1997). Agreeableness: A dimension of personality. In R. Hogan, J. Johnston, \& S. Briggs (Eds.), Handbook of personality psychology (pp. 795824). San Diego, CA: Academic Press.

Herzog, H. (2014, December 2). 84\% of vegetarians and vegans return to meat. Why? Psychology Today. Retrieved from https://www.psychologytoday.com/us/blog/animals-and-us/201412/84-vegetarians-andvegans-return-meat-why

Hornsey, M. J., \& Jetten, J. (2003). Not being what you claim to be: Impostors as sources of group threat. European Journal of Social Psychology, 33, 639-657.

Izmirli, S., \& Phillips, C. J. (2011). The relationship between student consumption of animal products and attitudes to animals in Europe and Asia. British Food Journal, 113, 436-450.

Jabs, J., Devine, C. M., \& Sobal, J. (1998). Model of the process of adopting vegetarian diets: Health vegetarians and ethical vegetarians. Journal of Nutrition Education, 30, 196-202.

Jabs, J., Sobal, J., \& Devine, C. M. (2000). Managing vegetarianism: Identities, norms and interactions. Ecology of Food and Nutrition, 39, 375-394.

Kessler, C. S., Holler, S., Joy, S., Dhruva, A., Michalsen, A., Dobos, G., \& Cramer, H. (2016). Personality profiles, values and empathy: Differences between lacto-ovo-vegetarians and vegans. Complementary Medicine Research, 23, 95-102. 
Kwan, S., \& Roth, L. (2004). Meat consumption and its discontents: Vegetarianism as counterhegemonic embodiment. In Conference Papers-American Sociological Association (pp. $1-14)$.

Lecky, P. (1945). Self-consistency: A theory of personality. New York: Island.

LeRette, D. E. (2014). Stories of microaggressions directed toward vegans and vegetarians in social settings (Doctoral dissertation, Fielding Graduate University).

Luhtanen, R., \& Crocker, J. (1992). A collective self-esteem scale: Self-evaluation of one's social identity. Personality and Social Psychology Bulletin, 18, 302-318.

Lund, T. B., McKeegan, D. E., Cribbin, C., \& Sandøe, P. (2016). Animal ethics profiling of vegetarians, vegans and meat-eaters. Anthrozoös, 29, 89-106.

MacInnis, C. C., \& Hodson, G. (2017). It ain’t easy eating greens: Evidence of bias toward vegetarians and vegans from both source and target. Group Processes \& Intergroup Relations, 20, 721-744.

Minson, J. A., \& Monin, B. (2012). Do-gooder derogation: Disparaging morally motivated minorities to defuse anticipated reproach. Social Psychological and Personality Science, 3, 200-207.

Monin, B. (2007). Holier than me? Threatening social comparison in the moral domain. Revue Internationale de Psychologie Sociale, 20, 53-68.

Ogden, J., Karim, L., Choudry, A., \& Brown, K. (2006). Understanding successful behaviour change: The role of intentions, attitudes to the target and motivations and the example of diet. Health Education Research, 22, 397-405.

Quinn, D. M., \& Chaudoir, S. R. (2009). Living with a concealable stigmatized identity: The impact of anticipated stigma, centrality, salience, and cultural stigma on psychological 
distress and health. Journal of Personality and Social Psychology, 97, 634-651.

Rosenberg, M., Schooler, C., Schoenbach, C., \& Rosenberg, F. (1995). Global self-esteem and specific self-esteem: Different concepts, different outcomes. American Sociological Review, 60, 141-156.

Rosenfeld, D. L. (2018). The psychology of vegetarianism: Recent advances and future directions. Appetite, 131, 125-138.

Rosenfeld, D. L. (2019a). A comparison of dietarian identity profiles between vegetarians and vegans. Food Quality and Preference, 72, 40-44.

Rosenfeld, D. L. (2019b). Gender differences in vegetarian identity: How men and women construe meatless dieting. Manuscript submitted for publication.

Rosenfeld, D. L. (2019c). Psychometric properties of the Dietarian Identity Questionnaire among vegetarians. Food Quality and Preference, 74, 135-141.

Rosenfeld, D. L. (2019d). Why some choose the vegetarian option: Are all ethical motivations the same? Motivation and Emotion, 43, 400-411.

Rosenfeld, D. L., \& Burrow, A. L. (2017a). The unified model of vegetarian identity: A conceptual framework for understanding plant-based food choices. Appetite, 112, 78-95.

Rosenfeld, D. L., \& Burrow, A. L. (2017b). Vegetarian on purpose: Understanding the motivations of plant-based dieters. Appetite, 116, 456-463.

Rosenfeld, D. L., \& Burrow, A. L. (2018). Development and validation of the Dietarian Identity Questionnaire: Assessing self-perceptions of animal-product consumption. Appetite, 127, 182-194.

Rosenfeld, D. L., \& Tomiyama, A. J. (2020). Taste and health concerns trump anticipated stigma as barriers to vegetarianism. Appetite, 144, 104469. 
Rothgerber, H. (2014a). A comparison of attitudes toward meat and animals among strict and semi-vegetarians. Appetite, 72, 98-105.

Rothgerber, H. (2014b). Evaluation of ingroup disloyalty within a multigroup context. Social Psychology, 45, 382-390.

Rothgerber, H. (2015). Can you have your meat and eat it too? Conscientious omnivores, vegetarians, and adherence to diet. Appetite, 84, 196-203.

Rothgerber, H. (2017). Attitudes toward meat and plants in vegetarians. In F. Mariotti (Ed.), Vegetarian and Plant-Based Diets in Health and Disease Prevention (pp. 11-35). London, UK: Academic Press.

Rozin, P., \& Fallon, A. E. (1987). A perspective on disgust. Psychological Review, 94, 2341.

Rozin, P., Markwith, M., \& Stoess, C. (1997). Moralization and becoming a vegetarian: The transformation of preferences into values and the recruitment of disgust. Psychological Science, 8, 67-73.

Sedupane, G. (2017). A qualitative study exploring the experiences of Black South African vegetarians residing in the urban settings of Cape Town (Master's thesis, University of Western Cape.

Sobal, J. (2005). Men, meat, and marriage: Models of masculinity. Food and Foodways, 13, 135158.

Stiles, B. (1998). Vegetarianism: Identity and experiences as factors in food selection. Free Inquiry in Creative Sociology, 26, 213-226.

Stryker, S., \& Serpe, R. T. (1982). Commitment, identity salience, and role behavior. In W. Ickes \& E. Knowles (Eds.), Personality, roles, and social behavior (pp. 199-218). New York: Springer-Verlag. 
Tajfel, H., \& Turner, J. C. (1985). The social identity theory of intergroup behavior. In Worchel, S., \& Austin, W. G. Eds. Psychology of Intergroup Relations. Vol. 2 (pp.7-24). Chicago: Nelson-Hall.

The Vegetarian Resource Group. (2016). How many adults in the U.S. are vegetarian and vegan? Retrieved from http://www.vrg.org/nutshell/Polls/2016_adults_veg.htm

Torti, J. M. (2017). The social and psychological well-being of vegetarians: A focused ethnography (Doctoral dissertation, University of Alberta).

Vartanian, L. R. (2015). Impression management and food intake. Current directions in research. Appetite, 86, 74-80.

White, R. F., Seymour, J., \& Frank, E. (1999). Vegetarianism among US women physicians. Journal of the American Dietetic Association, 99, 595-598.

Willett, W., Rockström, J., Loken, B., Springmann, M., Lang, T., Vermeulen, S., ... \& Jonell, M. (2019). Food in the Anthropocene: the EAT-Lancet Commission on healthy diets from sustainable food systems. The Lancet. https://doi.org/10.1016/S0140-6736(18)31788-4

Willetts, A. (1997). Bacon sandwiches got the better of me: Meat eating and vegetarianism in South East London. In Caplan, P. (Ed.), Food, Health and Identity (pp. 111-130). London, England: Routledge.

Yeh, H. Y. (2014). Voice with every bite: Dietary identity and vegetarians' "the-second-best" boundary work. Food, Culture \& Society, 17, 591-613. 